\title{
Palmprint Image Acquisition and Analysis System Based on IoT Technology
}

\author{
Zhiheng Zhu, Xinwu Chen, Youchao Tu, Xiaoling Zhang \\ School of Physics and Electronic Engineering, Xinyang Normal University, Xinyang, China \\ Email: chenxinwu@126.com
}

How to cite this paper: Zhu, Z.H., Chen, X.W., Tu, Y.C. and Zhang, X.L. (2020) Palmprint Image Acquisition and Analysis System Based on IoT Technology. Open Access Library Journal, 7: e6897. https://doi.org/10.4236/oalib.1106897

Received: October 13, 2020

Accepted: November 10, 2020

Published: November 13, 2020

Copyright () 2020 by author(s) and Open Access Library Inc.

This work is licensed under the Creative Commons Attribution International License (CC BY 4.0).

http://creativecommons.org/licenses/by/4.0/

\section{(c) (i) Open Access}

\begin{abstract}
With the rapid development and advancement of society, information technology represented by biometric recognition has developed rapidly. Palmprint images have a larger area, more texture details, rich texture features and distinguishable information, and palmprint recognition is opposite to the palm. The pattern image acquisition equipment and resolution requirements are not high, which is suitable for palmprint image acquisition and analysis under the framework of the Internet of Things (IoT), and then realize the design of recognition technology and system based on palmprint texture characteristics. The palmprint texture feature extraction and analysis algorithm is deployed on the cloud server, and the analysis result is fed back to the on-site collection device to realize the collection and analysis of palmprint images.
\end{abstract}

\section{Subject Areas}

Technology

\section{Keywords}

Image, IoT, Palmprint Texture, Feature Extraction, Multi-Scale, System Design

\section{Background}

In recent years, with the rapid development of technologies such as the IoT, artificial intelligence, and virtual reality, the rapid development of electronic information technology and network communication technology has been promoted. The palmprint image represented by biometric technology has stability, uniqueness and convenience characteristics, and has been widely used [1] [2] [3] [4] [5]. As a new biometric technology, palmprint texture recognition has the characteristics of simple sampling and rich image information. It has been fully 
applied in various fields, especially in the field of information security and health analysis combined with traditional Chinese medicine hand diagnosis [6].

Palm prints refer to the various lines on the surface of the palm between the wrist and fingers [7] [8]. Everyone's palmprint lines and texture features are different. The rich texture features in palmprint images contain a lot of body feedback information. Combining with the hand diagnosis theory of Chinese medicine, it combines the texture characteristics of palmprint with Chinese medicine. Hand diagnosis in traditional Chinese medicine is a medical-assisted diagnosis approach and treatment method. By observing the texture depth, color, contour shape and other texture characteristics of the palmprint, the patient's health status can be diagnosed [9] [10].

In order to quickly and accurately obtain palmprint texture feature information, the palmprint image acquisition and analysis system based on the IoT technology can achieve the following functions: 1) Collection and storage of massive palmprint images; 2) Using the feature database, algorithm can give guidelines to the users; 3) Appending palmprints for charactering new or unknown diseases for further research; 4) Promotion applying parmprint technology in daily life.

The palmprint image acquisition and analysis system studied in this paper is mainly composed of hardware system and software system. The hardware system is mainly composed of functional modules such as collecting, storing and uploading images, information processing, and human-computer interaction units. The software system is mainly composed of pre-processing, task algorithm, data processing, interactive processing and other main systems. The schematic diagram of the system architecture is shown in Figure 1.

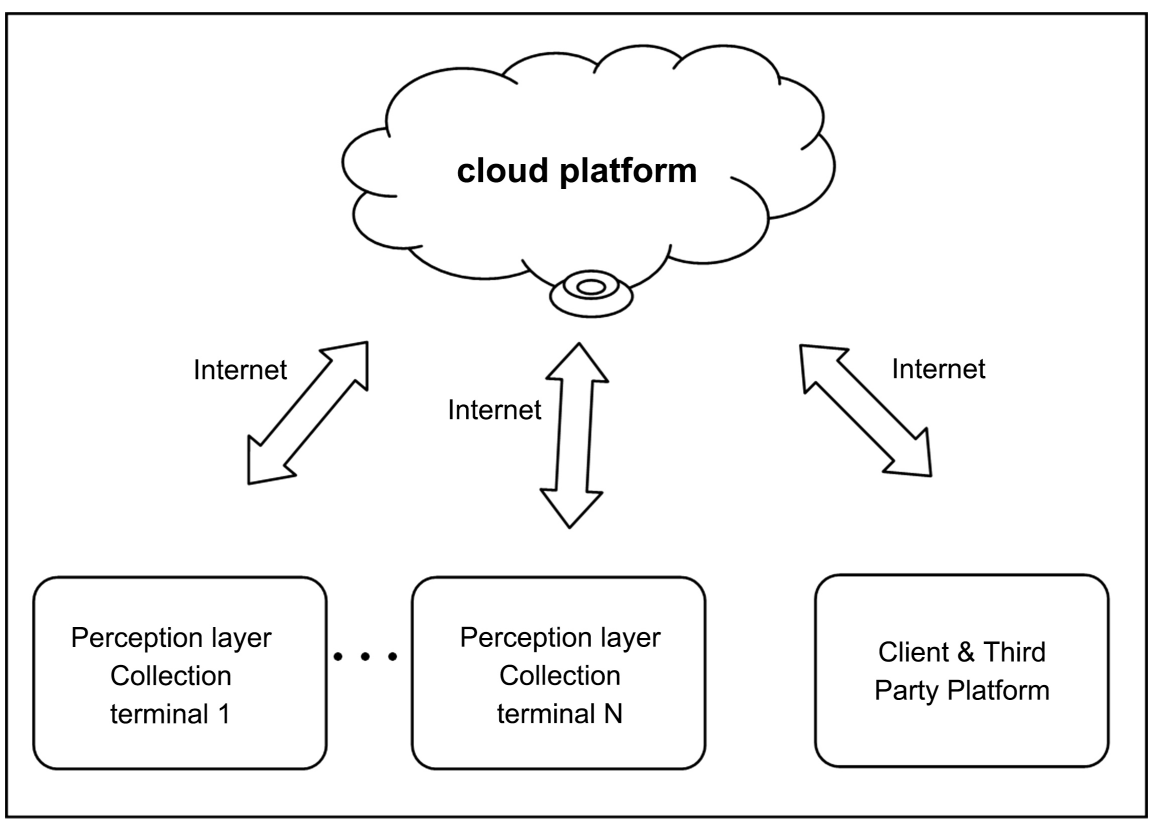

Figure 1. Schematic diagram of palmprint image acquisition and analysis system architecture based on IoT technology. 


\section{Hardware System}

The hardware system mainly includes power supply unit, central processing unit, information acquisition unit, data processing analysis unit, algorithm analysis unit, information reporting and receiving unit, display unit, etc. The schematic diagram of the hardware system structure is shown in Figure 2.

The power supply unit has the function of power management. It can be powered by mains AC220V and has a backup power supply. It can also be powered by a DC9-30V portable power supply (such as storage batteries, lithium batteries, etc.). In special scenarios, it can work normally for 48 - 72 hours. In the non-working state, the device is in a low power consumption mode, which greatly reduces the use of energy consumption. At the same time, the working status of the power management unit can be identified and monitored by the central processing unit, and reported and stored. Furthermore, the operation and maintenance analysis platform can grasp the working status of the power supply of the entire device in time.

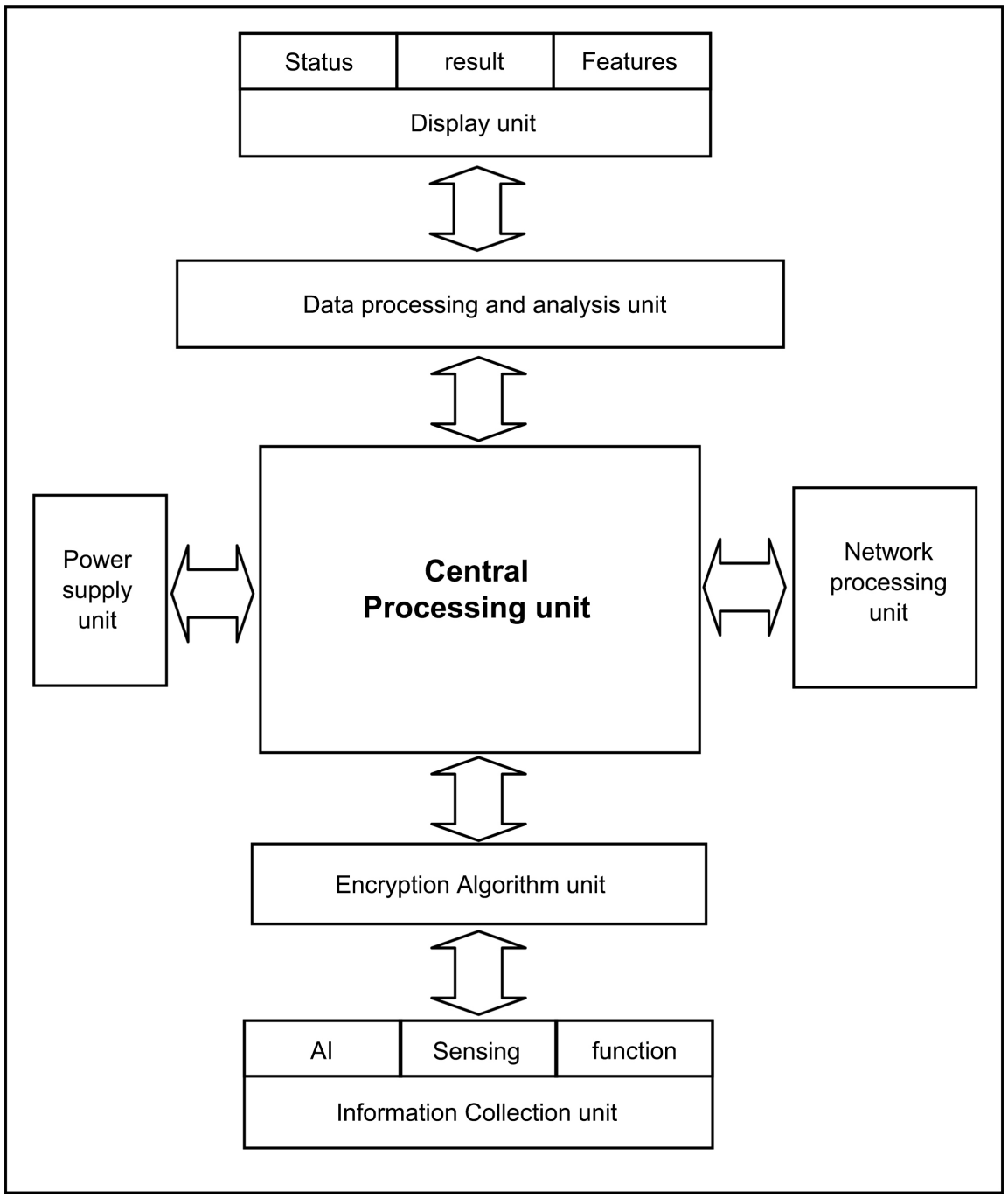

Figure 2. Schematic diagram of hardware system structure. 
The central processing unit is composed of programmable processors, including but not limited to STM32 series, ARM series, FPGA series and other processors, capable of fast execution of program codes, mainly for data algorithm analysis, data information processing, and display control of the display unit It is the core software operating unit of the entire device.

The information collection unit is composed of a peripheral collection device and a collection interface. The peripheral collection device can be a camera, a contact scanner, a two-dimensional or three-dimensional scanner and other devices that can obtain image information. The peripheral collection device collects the most primitive. The palmprint texture information is transmitted to the data processing and analysis unit through the acquisition interface.

The information collection unit collects the original palmprint texture information, and transmits the data to the data processing and analysis unit through the collection interface; the collection device can be a USB external image acquisition device, a two-dimensional or three-dimensional scan input device, or a piece of The complete palmprint picture is uploaded to the device; in short, the acquisition device can obtain clear and undistorted palmprint information, and can pass the graphic information into the device, and then perform feature extraction, system analysis, and display prompts.

The algorithm analysis unit analyzes the texture characteristic algorithm and gives the display result. The information sending and receiving unit is mainly composed of 3/4/5G network, WiFi, Internet, NB-IOT and other networks, which can realize the rapid, stable and accurate interaction of information; send field device collected information and characteristic information to the cloud Platform; Receive cloud platform response information and instructions, and perform data storage and push.

The display unit consists of a 7-inch or 10-inch touch LCD screen, which can run Android or iOS operating systems, and is responsible for the information display of the device and the information interaction with the user.

\section{Software System}

The software system is mainly composed of main system modules such as pre-service, task algorithm service, data processing service, and interactive processing program. The main function flowchart of the server is shown in Figure 3.

The software system mainly focuses on listening to the collected image information and performing multi-scale morphological algorithm analysis. The algorithm first converts palm prints into digital texture images. For digital texture images, first performs brightness regulation, interest area capture, and image matching. Quasi-equal preprocessing, and then perform complex contourlet transformation, calculate statistical features of the data in each sub-band, obtain transform domain features, construct multi-scale and multi-directional local texture information description operators in the spatial domain, and fuse them with transform domain features Obtain the digital feature of the palm print, store the feature in the database, and feed the result back to the field device. 


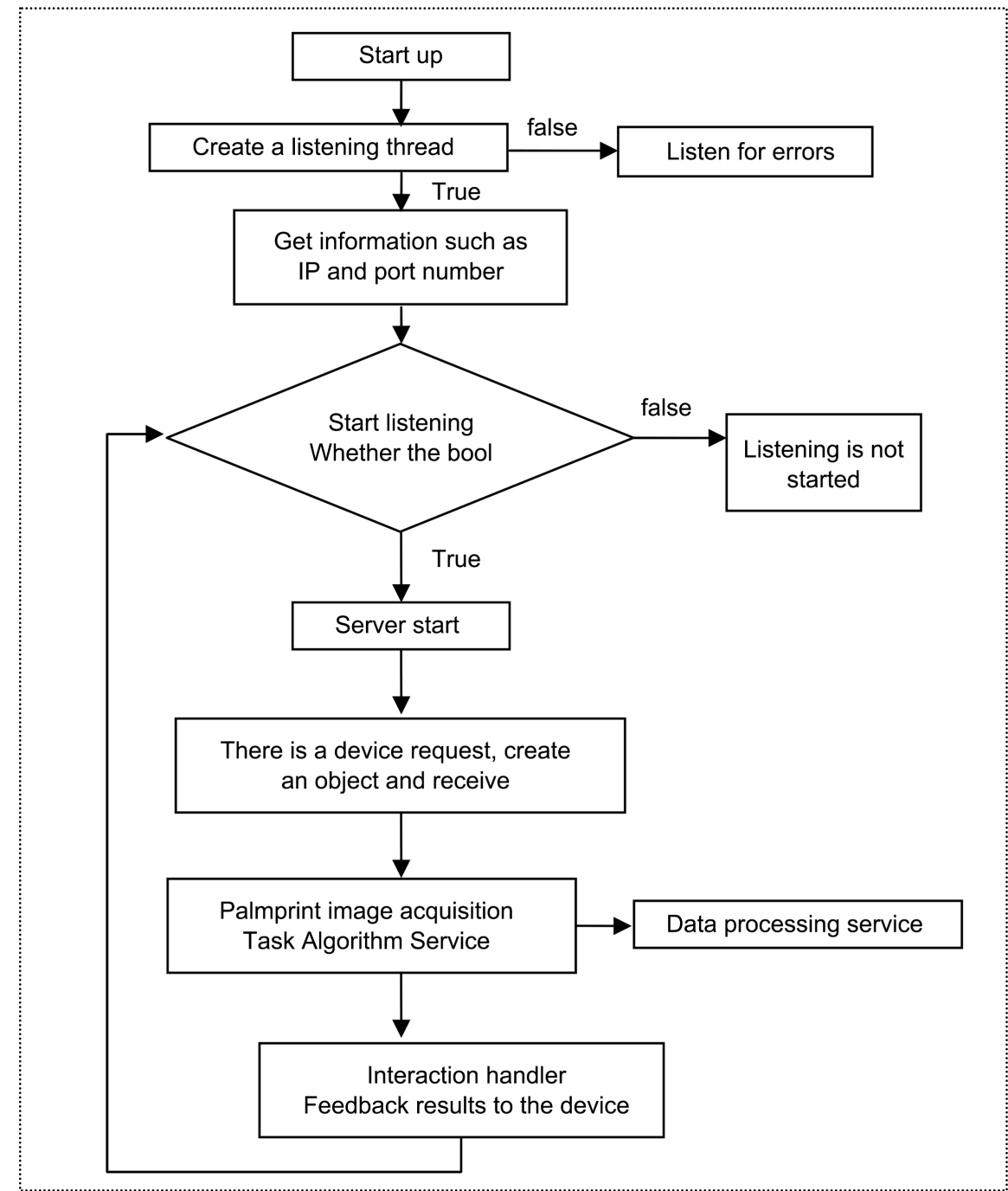

Figure 3. Server main function flowchart.

\section{Analysis of Algorithms}

The algorithm analysis system is deployed on the cloud server in the software system, and the cloud server performs algorithm analysis. Generally speaking, palmprint texture characteristics refer to the repetitive pattern of local changes in image gray levels. Palmprint texture is composed of texture primitives arranged in a certain pattern. The edge frequency method can measure the roughness of the texture or the complexity of the texture in the total number of edge points in a region. Because the texture has scale correlation, the Sobel or Robert edge detection operator with variable scale can be used to detect the short edge or long edge of the texture image.

The Fourier transform is a bridge between the time domain and the frequency domain, which provides the possibility for frequency domain analysis. Texture features are not only displayed in the time domain, but also in the frequency domain. For an $\mathrm{M}^{\star} \mathrm{N}$ two-dimensional digital image $f(x, y)$, its two-dimensional discrete Fourier transform is: 


$$
\begin{aligned}
& F(u, v)=\frac{1}{M N} \sum_{x=0}^{M-1} \sum_{y=0}^{N-1} f(x, y) \exp \left(-j 2 \pi\left(\frac{u x}{M}+\frac{v y}{N}\right)\right), \\
& u=0,1,2, \cdots, M-1 ; v=0,1,2, \cdots, N-1
\end{aligned}
$$

The corresponding inverse Fourier transform is:

$$
\begin{aligned}
& f(x, y)=\sum_{x=0}^{M-1} \sum_{y=0}^{N-1} F(u, v) \exp \left(j 2 \pi\left(\frac{u x}{M}+\frac{u y}{N}\right)\right), \\
& x=0,1,2, \cdots, M-1 ; \quad y=0,1,2, \cdots, N-1
\end{aligned}
$$

The available power spectrum is:

$$
P(u, v)=|F(u, v)|^{2}
$$

It can be seen from the nature of the Fourier transform that for large-sized texture primitives, the energy is mainly distributed in the low frequency band, while for small-sized texture primitives, the energy is mainly distributed in the high frequency band.

The possible frequency decomposition of contourlet transform is shown in Figure 4, and the decomposition of angular resolution can be carried out on different scales or directions. Using the wavelet transform implemented by the Contourlet transform tool, the palmprint feature image is shown in Figure 5.

In the algorithm analysis system, the feature data vectors of different scales and different frequency domains are extracted, and then retrieved with the feature vector of the feature database, and then the analysis result is given.

\section{Summary}

This article describes the hardware design and software design ideas of the palmprint image acquisition and analysis system based on the IoT technology, and gives the design process. Through experiments, the collection and analysis, upload and storage of palmprint images can be completed. The experimental results are shown in Figure 6.

The system completes the design of the hardware and software system framework. The service software and algorithm analysis components deployed on the cloud server can extract the texture of the palmprint texture image and form a feature vector library for analysis and storage, and optimize the feature algorithm model for further research. The popularization and application of palmprint images in real life has laid a certain foundation.
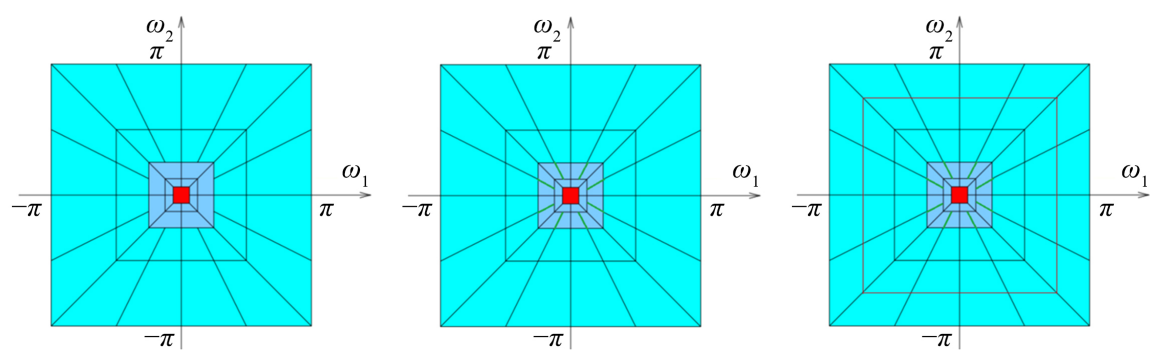

Figure 4. Schematic diagram of possible frequency decomposition of contour wave conversion. 


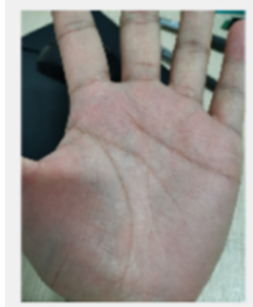

(a)

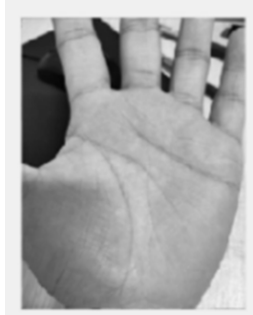

(d)

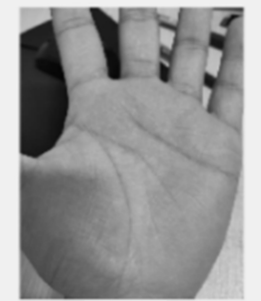

(b)

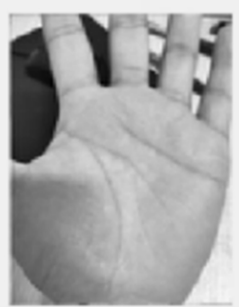

(e)

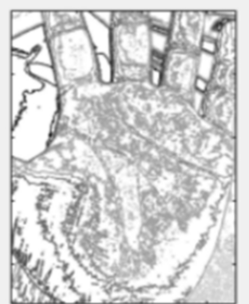

(c)

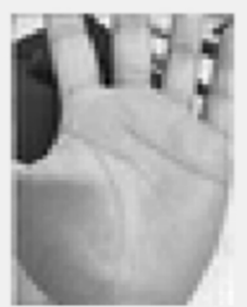

(f)

Figure 5. Palmprint image transformed by contourlet.

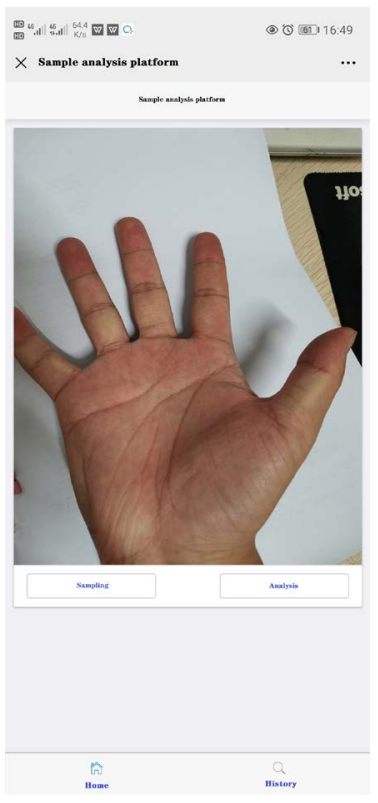

(a)

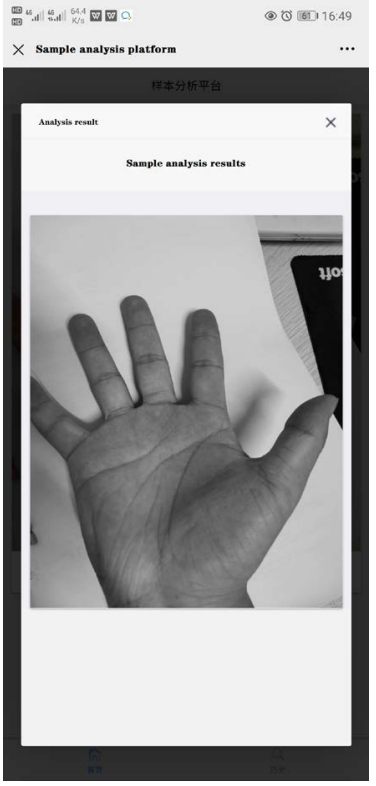

(b)

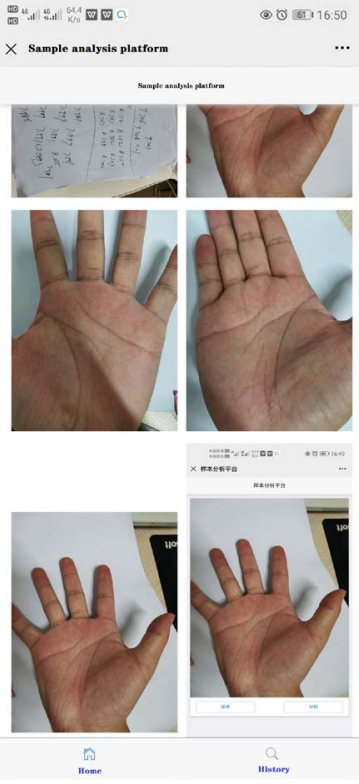

(c)

Figure 6. Palmprint image collection and analysis results. (a) Palm print collection; (b) Palmprint analysis; (c) History information.

\section{Acknowledgements}

This paper was supported by 2020 Henan Provincial University National College Student Innovation and Entrepreneurship Training Program Project (ID.202010477059S).

\section{Conflicts of Interest}

The authors declare no conflicts of interest regarding the publication of this paper. 


\section{References}

[1] Kolivand, H., Fern, B.M., Saba, T., Rahim, M.S.M. and Rehman, A. (2019) A New Leaf Venation Detection Technique for Plant Species Classification. Arabian Journal for Science and Engineering, 44, 3315-3327. https://doi.org/10.1007/s13369-018-3504-8

[2] Voorhees, H. and Poggio, T. (1987) Detecting Textons and Texture Boundaries in Natural Images. Proceedings of the First International Conference on Computer Vision, London, 8-11 June 1987, 250-258.

[3] Tomita, F. and Tsuji, S. (1990) Computer Analysis of Visual Textures. Springer International. https://doi.org/10.1007/978-1-4613-1553-7

[4] Haralick, R.M., Shanmugam, K. and Dinstein, I.H. (1973) Textural Features for Image Classification. IEEE Transactions on SMC, 3, 610-621.

https://doi.org/10.1109/TSMC.1973.4309314

[5] Srinivasa, G.N. and Shobba, G. (2006) Statistical Texture Analysis. Proceedings of World Academy of Science, Engineering and Technology, 11, 196-201.

[6] Bingöl, Ö. and Ekinci, M. (2017) Stereo-Based Palmprint Recognition in Various 3D Ostures. Expert Systems with Applications, 78, 74-88. https://doi.org/10.1016/j.eswa.2017.01.025

[7] Tabejamaat, M. and Mousavi, A. (2018) Generalized Gabor Filters for Palmprint Recognition. Pattern Analysis \& Applications, 21, 261-275. https://doi.org/10.1007/s10044-017-0638-3

[8] Pooniaa, P. and Ajmerab, P.K. (2020) Vijayendra Shendec. Palmprint Recognition Using Robust Template Matching. Procedia Computer Science, 167, 727-736. https://doi.org/10.1016/j.procs.2020.03.338

[9] Zhong, D., Du, X. and Zhong, K. (2019) Decade Progress of Palmprint Recognition: A Brief Survey (Article). Neurocomputing, 328, 16-28. https://doi.org/10.1016/j.neucom.2018.03.081

[10] Hao, F., Chang, X., Yang, G., Yang, L., Li, C., Li, C. and Xia, C. (2020) Local Image Quality Measurement for Multi-Scale Forensic Palmprints. Multimedia Tools and Applications, 79, 12915-12938. https://doi.org/10.1007/s11042-020-08625-y 